\title{
On the Multispecies Delayed Gurtin-MacCamy Model
}

\author{
Anna Poskrobko' and Antoni Leon Dawidowicz ${ }^{2}$ \\ ${ }^{1}$ Faculty of Computer Science, Bialystok University of Technology, Ulica Wiejska 45A, 15-351 Białystok, Poland \\ ${ }^{2}$ Faculty of Mathematics and Computer Science, Jagiellonian University, Ulica Łojasiewicza 6, 30-348 Kraków, Poland \\ Correspondence should be addressed to Anna Poskrobko; a.poskrobko@pb.edu.pl
}

Received 8 November 2012; Revised 9 March 2013; Accepted 29 March 2013

Academic Editor: Carlos Vazquez

Copyright ( 2013 A. Poskrobko and A. L. Dawidowicz. This is an open access article distributed under the Creative Commons Attribution License, which permits unrestricted use, distribution, and reproduction in any medium, provided the original work is properly cited.

The paper deals with the description of multispecies model with delayed dependence on the size of population. It is based on the Gurtin and MacCamy model. The existence and uniqueness of the solution for the new problem of $n$ populations dynamics are proved, as well as the asymptotical stability of the equilibrium age distribution.

\section{Introduction}

In this paper we consider mutual influence of $n$ populations. We assume that each population develops differently, affecting each other. Populations do not destroy each other. However, they share the same natural resources and the space. In our model $u$ denotes the density of the ecosystem consisting of $n$ different populations. Therefore $u=\left(u^{1}, \ldots, u^{n}\right)$ is the vector function. Each component $u^{i}$ for $i=1, \ldots, n$ denotes the $i$ th population density. The development of $i$ th population can be expressed by the following system of the equations:

$$
\begin{gathered}
D u^{i}(a, t)=-\lambda^{i}\left(a, z_{t}\right) u^{i}(a, t) \\
u^{i}(0, t)=\int_{0}^{\infty} \int_{[-r, 0]} \beta^{i}(a, z(t+s)) u^{i}\left(a, t+s^{i}\right) d s d a \\
z^{i}(t)=\int_{0}^{\infty} u^{i}(a, t) d a
\end{gathered}
$$

with the initial condition

$$
u^{i}(a, s)=\varphi^{i}(a, s) \quad \text { for } s \in\left[-r^{i}, 0\right]
$$

where

$$
\begin{gathered}
{[-r, 0]=\left[-r^{1}, 0\right] \times\left[-r^{2}, 0\right] \times \cdots \times\left[-r^{n}, 0\right],} \\
z(t+s)=\left(z^{1}\left(t+s^{1}\right), z^{2}\left(t+s^{2}\right), \ldots, z^{n}\left(t+s^{n}\right)\right), \\
z_{t}^{i}:\left[-r^{i}, 0\right] \longrightarrow \mathbb{R}_{+}, \quad r^{i} \geqslant 0, \mathbb{R}_{+}=[0, \infty), \\
z_{t}^{i}(s)=z^{i}(t+s) .
\end{gathered}
$$

The rate

$$
D u^{i}(a, t)=\lim _{h \rightarrow 0} \frac{u^{i}(a+h, t+h)-u^{i}(a, t)}{h}
$$

denotes the intensity of changing of the $i$ th population in time. In particular, if $u^{i}$ is differentiable then $D u^{i}=\partial u^{i} / \partial a+$ $\partial u^{i} / \partial t$. The quantity $z^{i}(t)$ is the total $i$ th population at time $t$. We can express total population of the whole ecosystem at time $t$ by

$$
z(t)=\left(z^{1}(t), \ldots, z^{n}(t)\right)
$$

Thus

$$
z_{t}=\left(z_{t}^{1}, \ldots, z_{t}^{n}\right)
$$


We assume that

$$
z_{0}^{i}(0)=\int_{0}^{\infty} \varphi^{i}(a, 0) d a .
$$

The birth and death processes of the $i$ th population are described by coefficients $\lambda^{i}$ and $\beta^{i}$. We assume that both processes depend on the population size not only at the moment $t$, but also at any preceding period of time (so-called history segment). Introducing the delay parameter to the model has deep biological approach (see, for instance, [1]). All natural processes occur with some delay with respect to the moment of their initiation. We can take into consideration, for example, the period of pregnancy (constant delay) or morbidity (variable delay). In our model, considered processes occur with $n$ various delays, typical for $n$ populations. It is a novel approach to the population dynamics research. Moreover the system (1)-(4) consists of the general description of $n$ populations dynamics. If populations develop independently, then the functions $\lambda^{i}$ and $\beta^{i}$ depend on $i$ th coordinate of the function $z_{t}$ only and the system consists of $n$ independent von Foerster equations. However, if there are populations relationships, we can consider three cases.

(1) Competition for food: in this case $\beta^{i}$ is decreasing function of variables describing the numbers of species competing for food with the $i$ th one. For example, $\beta^{i}(a, z)=\max \left\{a\left(F-\sum_{j \in I} k_{j} z_{j}\right), 0\right\}$, where $F$ denotes total amount of food and $I$ is the set of species competing with the $i$ th one.

(2) Schema predator prey: if $i$ th species feeds on $j$ th species individuals, then $\lambda^{j}$ and $\beta^{i}$ are increasing functions of variables $z^{j}$ and $z^{i}$, respectively. The classical models show that balance state between two species competing for food is not possible. In this case the model does not have nonzero equilibrium age distribution. However, if we additionally consider predator feeding on one competing species, then we get balance between three considered species (see, for instance, [2-5]).

(3) Symbiosis: if $i$ th and $j$ th species live in symbiosis with each other, then $\beta^{i}$ can be an increasing function of $z^{j}$ (when the presence of $j$ th species individuals is the reproduction of $i$ th species favour) or $\lambda^{j}$ is a decreasing function of $z^{i}$ (when the presence of $i$ th species protects $j$ th species individuals from death).

In this paper we develop the idea of Gurtin and MacCamy model [6] for one age-dependent population

$$
\begin{gathered}
D u(a, t)=-\lambda(a, z(t)) u(a, t), \\
u(0, t)=\int_{0}^{\infty} \beta(a, z(t)) u(a, t) d a, \\
z(t)=\int_{0}^{\infty} u(a, t) d a, \\
u(a, 0)=\varphi(a) .
\end{gathered}
$$

This classical model has many generalizations [7-16]. Considerations of the dynamics of age-structured populations have received substantial treatment on various fields [1722]. In particular our latest extension of this theme was presented in the article [10], where we considered an agedependent population dynamics with a delayed dependence on the structure. The right-hand side of the equation in our model is not in the form $\lambda u$ but $\Lambda u$. Here $\Lambda$ is an operator that does not apply to the value of the function $u$, as in the classical model, but to its restriction to some particular space:

$$
\begin{gathered}
D u(a, t)=-\Lambda\left(a, z_{t}\right) u(a, t+\cdot), \\
u(0, t)=\int_{0}^{\infty} \int_{-r}^{0} \beta(a, z(t+s)) u(a, t+s) d s d a, \\
z(t)=\int_{0}^{\infty} u(a, t) d a \quad \text { for } t \in[-r, T] \\
u(a, s)=\varphi(a, s) \quad \text { for } s \in[-r, 0] .
\end{gathered}
$$

In our paper [10] we present the proof of the existence and uniqueness of the new problem solution. Conditions of the exponential asymptotical stability for this model still remain to be formulated. On account of the significant difficulties with formulating conditions of the stability we consider slightly simpler version of the model than the one described in [10]. We resign from the delay in the structure during a deliberation on mortality. However, the delayed dependence of the structure still remains the element of the birth process description. In this paper we aim to state stability conditions for the ecosystem of $n$ populations. The plan of the paper is as follows. In Section 2 we formulate the problem in the terms of operator equations. Section 3 contains the proof of the existence and uniqueness of the solution. We also study equilibrium age distributions, that is, solutions to the problem which are independent of time. In Section 4 exponential asymptotic stability of the equilibrium age distributions is analyzed. We use generalized Laplace transform to study the stability.

We consider our model (1)-(4) under the following assumptions.

$\left(H_{1}\right) \varphi=\left(\varphi^{1}, \ldots, \varphi^{n}\right)$ where $\varphi^{i} \in C\left(\mathbb{R}_{+} \times\left[-r^{i}, 0\right]\right)$ for $i=$ $1, \ldots, n$.

$\left(H_{2}\right) \Phi^{i}=\int_{0}^{\infty} \sup _{s \in\left[-r^{i}, 0\right]} \varphi^{i}(a, s) d a<\infty$ for $i=1, \ldots, n$.

$\left(H_{3}\right)$ The function $\left[-r^{i}, 0\right] \ni t \mapsto \int_{0}^{\infty} \varphi^{i}(a, t) d a$ is continuous.

$\left(H_{4}\right) \lambda=\left(\lambda^{1}, \ldots, \lambda^{n}\right), \beta=\left(\beta^{1}, \ldots, \beta^{n}\right)$ where $\lambda^{i} \epsilon$ $C\left(\mathbb{R}_{+} \times \chi_{i=1}^{n} C\left(\left[-r^{i}, 0\right]\right)\right), \beta^{i} \in C\left(\mathbb{R}_{+}^{n+1}\right)$; the Fréchet derivatives $D \lambda^{i}$ of $\lambda^{i}(a, \psi)$ with respect to $\psi$ exist for all $a \geqslant 0$ and $\psi \geqslant 0$.

$\left(H_{5}\right)$ The components of the function $\lambda(\cdot, \psi)=\left(\lambda^{1}(\cdot, \psi)\right.$, $\left.\ldots, \lambda^{n}(\cdot, \psi)\right)$ belong to $C\left(C\left(\left[-r^{i}, 0\right]\right) ; L^{\infty}\left(\mathbb{R}_{+}\right)\right)$, respectively, for $i=1, \ldots, n$.

$\left(H_{6}\right)$ The components of Fréchet derivative $D_{\psi_{0}} \lambda=$ $\left(D_{\psi_{0}} \lambda^{1}, \ldots, D_{\psi_{0}} \lambda^{n}\right)$ in the point $\psi_{0}$ as functions of $\psi_{0}$ belong to $C\left(C\left(\left[-r^{i}, 0\right]\right) ; \mathscr{L}\left(C\left(\left[-r^{i}, 0\right]\right), L^{\infty}\left(\mathbb{R}_{+}\right)\right)\right)$, $i=1, \ldots, n$. Here $\mathscr{L}(X, Y)$ denotes the Banach space of all bounded linear operators from $X$ to $Y$. 
$\left(H_{7}\right) \varphi^{i} \geqslant 0, \lambda^{i} \geqslant 0, \beta^{i} \geqslant 0$ for $i=1, \ldots, n$.

$\left(H_{8}\right)$ The functions $\lambda$ and $\beta$ are bounded; that is, $\|\lambda(a, \psi)\| \leqslant \lambda_{0}$ and $\|\beta(a, z)\| \leqslant \beta_{0}$ where $\lambda_{0}$ and $\beta_{0}$ are finite quantities.

$\left(H_{9}\right)$ There exist $\lambda_{1}$ and $\beta_{1}$ such that

$$
\left\|\lambda\left(a, \psi_{1}\right)-\lambda\left(a, \psi_{2}\right)\right\| \leqslant \lambda_{1}\left\|\psi_{1}-\psi_{2}\right\|
$$

for every $a, \psi_{1}, \psi_{2}$ and

$$
\sum_{i=1}^{n} \sum_{j=1}^{n}\left|\frac{\partial \beta^{i}(a, z)}{\partial z^{j}}\right| \leqslant \beta_{1}
$$

for every $a, z$.

\section{Equivalent Formulation of the Problem}

In this section we will formulate problem (1)-(4) in terms of operator equations. Thanks to this we will prove local and global existences of the presented problem solution. The next theorem is analogous to these well-known results, for example, von Foerster [23] or Gurtin and MacCamy models.

Theorem 1. Nonnegative continuous functions $z_{t}=\left(z_{t}^{1}, \ldots\right.$, $\left.z_{t}^{n}\right)$ and $B=\left(B^{1}, \ldots, B^{n}\right)$, where

$$
\begin{aligned}
B^{i}(t)= & \int_{[-r, 0]} \int_{0}^{t+s^{i}} \beta^{i}\left(t+s^{i}-a, z(t+s)\right) \\
& \times B^{i}(a) e^{-\int_{a}^{t+s^{i}} \lambda^{i}\left(\tau-a, z_{\tau}\right) d \tau} d a d s \\
& +\int_{[-r, 0]} \int_{0}^{\infty} \beta^{i}\left(a+t+s^{i}, z(t+s)\right) \\
z_{t}^{i}\left(s^{i}\right)= & \times \varphi^{i}(a) e^{-\int_{0}^{t+s^{i}} \lambda^{i}\left(a+\tau, z_{\tau}\right) d \tau} d a d s, \\
& +\int_{0}^{t+s^{i}} B^{i}(a) e^{-\int_{a}^{t+s^{i}} \lambda^{i}\left(\tau-a, z_{\tau}\right) d \tau} d a \\
& z_{t}^{i}\left(s^{i}\right)=z_{0}^{i}\left(t+s^{i}\right) \quad \text { for } t+s^{i}<0,
\end{aligned}
$$

for $i=1, \ldots, n$, are the solutions of the problems (2) and (3) up to time $T>0$ if and only if the function $u=\left(u^{1}, \ldots, u^{n}\right)$ is the solution of the age-dependent $n$ populations problem (1)-(4) on $[0, T]$ and $u$ is defined by the formula

$$
u^{i}(a, t)= \begin{cases}\varphi^{i}(a-t, 0) e^{-\int_{0}^{t} \lambda^{i}\left(a-t+\tau, z_{\tau}\right) d \tau} & \text { for } a \geqslant t, \\ B^{i}(t-a) e^{-\int_{0}^{a} \lambda^{i}\left(\alpha, z_{t-a+\alpha}\right) d \alpha} & \text { for } t>a,\end{cases}
$$

where $B^{i}(t)=u^{i}(0, t)$ for each $i=1, \ldots, n$.

Proof. The idea of the proof is analogous to the result included in the paper [9], treating the theme of the age-dependent population problem for one species. Let $u=\left(u^{1}, \ldots, u^{n}\right)$ be a solution of the problem up to time $T$. Let $\bar{u}^{i}(h)=u^{i}\left(a_{0}+\right.$ $\left.h, t_{0}+h\right), \bar{\lambda}^{i}(h)=\lambda^{i}\left(a_{0}+h, z_{t_{0}+h}\right)$ then we can rewrite (1) as the equation $d \bar{u}^{i} / d h+\bar{\lambda}^{i}(h) \bar{u}^{i}=0$ with the unique solution

$$
u^{i}\left(a_{0}+h, t_{0}+h\right)=u^{i}\left(a_{0}, t_{0}\right) e^{-\int_{0}^{h} \bar{\lambda}^{i}(\eta) d \eta} .
$$

Substituting $\left(a_{0}, t_{0}\right)=(a-t, 0), h=t$ and $\left(a_{0}, t_{0}\right)=(0, t-a)$, $h=a$ into (17) yields the formula (16). Applying (16) to (2) and (3) we obtain the operator equations (14) and (15).

To prove the second part of the theorem we should assume that $z_{t}^{i} \geqslant 0$ and $B^{i} \geqslant 0$ for $i=1, \ldots, n$ are continuous functions on the interval $[0, T]$ fulfilling conditions (14) and (15). Let $u$ for each component $u^{i}$ be defined on $\mathbb{R}_{+} \times[0, T]$ by the formula (16). The function $u$ is nonnegative because of $\left(H_{7}\right)$. An easy computation shows that $(4)$ holds, and $u(0, t)=$ $B(t)$ for $t>0 . u \in L^{1}\left(\mathbb{R}_{+}\right)$because $\lambda, \beta$, and $z_{t}$ are continuous and $\varphi \in L^{1}(\mathbb{R})$. It follows from (14)-(16) that (2) and (3) are satisfied. To complete the proof, let us notice that (6) and (16) imply existing $D u$ on $\mathbb{R}_{+} \times[0, T]$ and the equality (1) holds.

Let us make some estimation for the necessity of the next section. We turn back to the operator equation (14). By the assumptions $\left(\mathrm{H}_{2}\right)$ and $\left(\mathrm{H}_{8}\right)$ we get

$$
B^{i}(t) \leqslant \beta_{0} r^{1} \cdots r^{n} \int_{0}^{t} \sup _{s \in\left[-r^{i}, 0\right]} B^{i}(a+s) d a+\beta_{0} r^{1} \cdots r^{n} \Phi^{i} .
$$

Denoting

$$
\mathscr{B}^{i}(t)=\sup _{\tau \in\left[-r^{i}, t\right]} B^{i}(\tau)
$$

we obtain

$$
\mathscr{B}^{i}(t) \leqslant \beta_{0} r^{1} \cdots r^{n} \int_{0}^{t} \mathscr{B}^{i}(a) d a+\beta_{0} r^{1} \cdots r^{n} \Phi^{i},
$$

and by Gronwall's inequality we get

$$
\mathscr{B}^{i}(t) \leqslant \beta_{0} r^{1} \cdots r^{n} \Phi^{i} e^{\beta_{0} r^{1} \cdots r^{n} t} .
$$

\section{Existence and Uniqueness}

According to the theorem in Section 2 to solve the population problem up to time $T$ it is sufficient to find functions $z=$ $\left(z^{1}, \ldots, z^{n}\right) \in X_{i=1}^{n} C^{+}\left(\left[-r^{i}, T\right]\right)$ and $B=\left(B^{1}, \ldots, B^{n}\right) \in$ $X_{i=1}^{n} C^{+}\left(\left[-r^{i}, T\right]\right)$ satisfying the system of operator equations (14) and (15). We can notice that (14) is a Volterra equation of $B^{i}$ with a unique solution $\mathscr{B}_{T}^{i}(z)$. Let us define on $X_{i=1}^{n} C^{+}\left(\left[-r^{i}, T\right]\right)$ the new operator $\mathscr{Z}_{T}=\left(\mathscr{Z}_{T}^{1}, \ldots, \mathscr{Z}_{T}^{n}\right)$ where

$$
\begin{aligned}
\mathscr{Z}_{T}^{i}(z)(t)= & \int_{0}^{t} \mathscr{B}_{T}^{i}(z)(a) e^{-\int_{a}^{t} \lambda^{i}\left(\tau-a, z_{\tau}\right) d \tau} d a \\
& +\int_{0}^{\infty} \varphi^{i}(a, 0) e^{-\int_{0}^{t} \lambda^{i}\left(a+\tau, z_{\tau}\right) d \tau} d a .
\end{aligned}
$$

We define the previous operator using the system of (15) for $i=1, \ldots, n$ with $B=\left(B^{1}, \ldots, B^{n}\right)$ replaced by $\mathscr{B}_{T}=$ $\left(\mathscr{B}_{T}^{1}, \ldots, \mathscr{B}_{T}^{n}\right)$. 
Theorem 2. The operator $\mathscr{Z}_{T}: X_{i=1}^{n} C^{+}\left[-r^{i}, T\right] \rightarrow$ $\mathrm{X}_{i=1}^{n} C^{+}\left[-r^{i}, T\right]$ defined by (22) has a unique fixed point for any $T>0$.

Proof. We prove that the operator $\mathscr{Z}_{T}$ is contracting, and then the assertion will be a consequence of the Banach fixed point theorem. Let us consider the Banach space $C\left[-r^{i}, T\right]$ with the Bielecki norm $\left\|f^{i}\right\|_{T}=\sup _{t \in\left[-r^{i}, T\right]} e^{-K t}\left|f^{i}(t)\right|$ for any $K>0$. Such norm is equivalent to classical supremum norm $\|\cdot\|$ in $C\left[-r^{i}, T\right]$. Choose $z, \widehat{z} \in X_{i=1}^{n} C\left[-r^{i}, T\right]$. Applying the definition (22) we obtain

$$
\begin{aligned}
& \left\|\mathscr{Z}_{T}^{i}(z)-\mathscr{Z}_{T}^{i}(\widehat{z})\right\|_{T} \\
& \leqslant \sup _{t \in\left[-r^{i}, T\right]} e^{-K t} \\
& \quad \times \int_{0}^{t}\left|e^{-\int_{a}^{t} \lambda^{i}\left(\tau-a, z_{\tau}\right) d \tau}-e^{-\int_{a}^{t} \lambda^{i}\left(\tau-a, \bar{z}_{\tau}\right) d \tau}\right| \mathscr{B}_{T}^{i}(z)(a) d a \\
& \quad+\sup _{t \in\left[-r^{i}, T\right]} e^{-K t} \\
& \quad \times \int_{0}^{t} e^{-\int_{a}^{t} \lambda^{i}\left(\tau-a, \widehat{z}_{\tau}\right) d \tau}\left|\mathscr{B}_{T}^{i}(z)(a)-\mathscr{B}_{T}^{i}(\widehat{z})(a)\right| d a \\
& \quad+\sup _{t \in\left[-r^{i}, T\right]} e^{-K t} \\
& \quad \times \int_{0}^{\infty}\left|e^{-\int_{0}^{t} \lambda^{i}\left(a+\tau, z_{\tau}\right) d \tau}-e^{-\int_{0}^{t} \lambda^{i}\left(a+\tau, \bar{z}_{\tau}\right) d \tau}\right| \varphi^{i}(a, 0) d a \\
& =I_{1}+I_{2}+I_{3} .
\end{aligned}
$$

Estimating the quantities $I_{1}, I_{2}$, and $I_{3}$ we use the assumptions $\left(H_{2}\right),\left(H_{7}\right)$, and $\left(H_{8}\right)$, inequalities (21) and $\left|e^{k}-1\right| \leqslant$ $|k| e^{|k|}$. Thus

$$
\begin{aligned}
I_{1} \leqslant & \sup _{t \in\left[-r^{i}, T\right]} e^{-K t} \\
& \times \int_{0}^{t}\left|1-e^{-\int_{a}^{t}\left[\lambda^{i}\left(\tau-a, z_{\tau}\right)-\lambda^{i}\left(\tau-a, \widehat{z}_{\tau}\right)\right] d \tau}\right| \mathscr{B}_{T}^{i}(z)(a) d a \\
\leqslant & \lambda_{1}\|z-\widehat{z}\|_{T} \sup _{t \in\left[-r^{i}, T\right]} e^{-K t} \\
& \times \int_{0}^{t} \int_{a}^{t} e^{2 \lambda_{0}(t-a)} e^{K \tau} \mathscr{B}_{T}^{i}(z)(a) d \tau d a \\
\leqslant & \frac{\lambda_{1}}{K} \frac{\beta_{0} r^{1} \cdots r^{n} \Phi^{i}}{\beta_{0} r^{1} \cdots r^{n}-2 \lambda_{0}} e^{\beta_{0} r^{1} \cdots r^{n} T}\|z-\widehat{z}\|_{T}, \\
I_{3} \leqslant & \sup _{t \in\left[-r^{i}, T\right]} e^{-K t} \\
& \times \int_{0}^{\infty} \int_{a}^{t} e^{2 \lambda_{0}(t-a)} e^{K \tau} \lambda_{1}\|z-\widehat{z}\|_{T} \varphi^{i}(a, 0) d \tau d a \\
\leqslant & \frac{\lambda_{1}}{K} \Phi^{i} e^{2 \lambda_{0} T}\|z-\widehat{z}\|_{T} .
\end{aligned}
$$

From (14) and the definition of $\mathscr{B}_{T}$ we have

$$
\begin{aligned}
& \mathscr{B}_{T}^{i}(z)(t)-\mathscr{B}_{T}^{i}(\widehat{z})(t) \\
& =\int_{[-r, 0]} \int_{0}^{t+s^{i}} \beta^{i}\left(t+s^{i}-a, z(t+s)\right) \cdot e^{-\int_{a}^{t+s^{i}} \lambda^{i}\left(\tau-a, z_{\tau}\right) d \tau} \quad(25) \\
& \times\left(\mathscr{B}_{T}^{i}(z)(a)-\mathscr{B}_{T}^{i}(\widehat{z})(a)\right) d a d s \\
& +\int_{[-r, 0]} \int_{0}^{t+s^{i}} \mathscr{B}_{T}^{i}(\widehat{z})(a) \\
& \times\left(\beta^{i}\left(t+s^{i}-a, z(t+s)\right)\right. \\
& \quad \times e^{-\int_{a}^{t+s^{i}} \lambda^{i}\left(\tau-a, z_{\tau}\right) d \tau}-\beta^{i}\left(t+s^{i}-a, \widehat{z}(t+s)\right) \\
& \left.\quad \times e^{-\int_{a}^{t+s^{i}} \lambda^{i}\left(\tau-a, \widehat{z}_{\tau}\right) d \tau}\right) d a d s \\
& +\int_{[-r, 0]}^{\infty} \int_{0}^{i}(a, 0) \\
& \times\left(\beta^{i}\left(a+t+s^{i}, z(t+s)\right)\right. \\
& \times e^{-\int_{0}^{t+s^{i}} \lambda^{i}\left(a+\tau, z_{\tau}\right) d \tau}-\beta^{i}\left(a+t+s^{i}, \widehat{z}(t+s)\right) \\
& \left.\times e^{-\int_{0}^{t+s^{i}} \lambda^{i}\left(a+\tau, \widehat{z}_{\tau}\right) d \tau}\right) d a d s .
\end{aligned}
$$

Let us denote (26) by $f^{i}(t)$; then

$$
\begin{aligned}
\mathscr{B}_{T}^{i}(z)(t)-\mathscr{B}_{T}^{i}(\widehat{z})(t) \\
\leqslant \beta_{0} r^{1} \cdots r^{n} \int_{0}^{t}\left(\mathscr{B}_{T}^{i}(z)(a)-\mathscr{B}_{T}^{i}(\widehat{z})(a)\right) d a \\
\quad+\left|f^{i}(t)\right|,
\end{aligned}
$$

and hence, by Gronwall's inequality we have

$$
\begin{aligned}
& \mathscr{B}_{T}^{i}(z)(t)-\mathscr{B}_{T}^{i}(\widehat{z})(t) \\
& \quad \leqslant\left|f^{i}(t)\right|+\beta_{0} r^{1} \cdots r^{n} \int_{0}^{t}\left|f^{i}(a)\right| e^{\beta_{0} r^{1} \cdots r^{n}(t-a)} d a .
\end{aligned}
$$

Let us estimate $\left|f^{i}(t)\right|$

$$
\begin{aligned}
& \left|f^{i}(t)\right| \\
& \leqslant \int_{[-r, 0]} \int_{0}^{t+s^{i}} C_{1} \mathscr{B}_{T}^{i}(\widehat{z})(a) \\
& \times\left(\beta^{i}\left(t+s^{i}-a, z(t+s)\right)\right. \\
& \left.-\beta^{i}\left(t+s^{i}-a, \widehat{z}(t+s)\right)\right) d a d s
\end{aligned}
$$




$$
\begin{aligned}
& +\int_{[-r, 0]} \int_{0}^{\infty} C_{2} \varphi^{i}(a, 0) \\
& \times\left(\beta^{i}\left(a+t+s^{i}, z(t+s)\right)\right. \\
& \left.-\beta^{i}\left(a+t+s^{i}, \widehat{z}(t+s)\right)\right) d a d s \\
& \leqslant \widetilde{C} \beta_{1} r^{1} \cdots r^{n} e^{K t}\|z-\widehat{z}\|_{T} \\
& \times\left(\Phi^{i} \int_{0}^{t} \beta_{0} r^{1} \cdots r^{n} e^{\beta_{0} r^{1} \cdots r^{n} a} d a+\int_{0}^{\infty} \varphi^{i}(a, 0) d a\right) \\
& \leqslant \widetilde{C} \beta_{1} r^{1} \cdots r^{n} \Phi^{i} e^{K t} e^{\beta_{0} r^{1} \cdots r^{n} t}\|z-\widehat{z}\|_{T},
\end{aligned}
$$

where $C_{1}$ and $C_{2}$ are positive constants and $\widetilde{C}=\max \left\{C_{1}, C_{2}\right\}$. Finally, we have

$$
\begin{aligned}
& I_{2} \leqslant \sup _{t \in\left[-r^{i}, T\right]} e^{-K t} \\
& \quad \times \int_{0}^{t}\left(\left|f^{i}(a)\right|+\beta_{0} r^{1} \cdots r^{n} \int_{0}^{a}\left|f^{i}(\xi)\right| e^{\beta_{0} r^{1} \cdots r^{n}(a-\xi)} d \xi\right) d a \\
& \leqslant \widetilde{C} \beta_{1} r^{1} \cdots r^{n} \Phi^{i} e^{K t}\|z-\widehat{z}\|_{T} \\
& \quad \times \sup _{t \in\left[-r^{i}, T\right]} e^{-K t}\left(\int_{0}^{t} e^{\left(K+\beta_{0} r^{1} \cdots r^{n}\right) a} d a\right. \\
& \left.\quad+\beta_{0} r^{1} \cdots r^{n} \int_{0}^{t} \int_{0}^{a} e^{K \xi} e^{\beta_{0} r^{1} \cdots r^{n} a} d \xi d a\right) \\
& \leqslant \\
& \quad \frac{\widetilde{C} \beta_{1} r^{1} \cdots r^{n} \Phi^{i}}{K+\beta_{0} r^{1} \cdots r^{n}}\|z-\widehat{z}\|_{T} e^{\beta_{0} r^{1} \cdots r^{n} T}\left(1+\frac{\beta_{0} r^{1} \cdots r^{n}}{K}\right) .
\end{aligned}
$$

Therefore, we can choose sufficiently large constant $K$ for fixed $T$ that $I_{1}, I_{2}$, and $I_{3}$ are less than $C\|z-\widehat{z}\|_{T}$ with the constant $C \in[0,(1 / 3 n)$ ) (independent of $z$ and $\widehat{z}$ ). This shows the contraction of $\mathscr{Z}_{T}$ and completes the proof.

According to the previous mentioned there exists the exact one solution for any interval $[0, T]$ so the solutions defined on two different intervals coincide on their intersection. By the extension property we have the existence and uniqueness of the $n$ populations problem solution for all times.

\section{Stability of Equilibrium Age Distribution}

A stationary solution $u(a)=\left(u^{1}(a), \ldots, u^{n}(a)\right)$ of the model (1)-(4) satisfies the following system of the equations:

$$
\begin{gathered}
\left(u_{0}^{i}\right)^{\prime}(a)+\lambda^{i}\left(a, z_{0}\right) u_{0}^{i}=0, \\
z_{0}^{i}=\int_{0}^{\infty} u_{0}^{i}(a) d a, \\
u_{0}^{i}(0)=r^{1} \cdots r^{n} \int_{0}^{\infty} \beta^{i}\left(a, z_{0}\right) u_{0}^{i}(a) d a
\end{gathered}
$$

for $i=1, \ldots, n$.
The population of the whole ecosystem $z_{0}=\left(z_{0}^{1}, \ldots, z_{0}^{n}\right)$ and its birthrate $B_{0}=u_{0}(0)=\left(u_{0}^{1}(0), \ldots, u_{0}^{n}(0)\right)$ are constants. The quantity $u_{0} \in C^{1}\left(\mathbb{R}_{+}\right)$is the solution of the system (31), and it will be referred to the equilibrium age distribution. The probability that an individual of $i$ th population survives to age $a$ if the population of the ecosystem is on the constant size level $z_{0}$ can be expressed by

$$
\pi_{0}^{i}(a)=e^{-\int_{0}^{a} \lambda^{i}\left(\alpha, z_{0}\right) d \alpha}
$$

The quantity

$$
R^{i}\left(z_{0}\right)=r^{1} \cdots r^{n} \int_{0}^{\infty} \beta^{i}\left(a, z_{0}\right) \pi_{0}^{i}(a) d a
$$

is the number of offsprings expected to be born to an individual of $i$ th population when the population of the whole ecosystem equals $z_{0}$. We can formulate the following theorem describing the connection between these three quantities.

Theorem 3. Let $z_{0}=\left(z_{0}^{1}, \ldots, z_{0}^{n}\right)$ and $z_{0}^{i}>0$ for $i=1, \ldots, n$, and assume that $\pi_{0}^{i}(\cdot), \beta^{i}\left(\cdot, z_{0}\right) \pi_{0}^{i}(\cdot) \in L^{1}\left(\mathbb{R}_{+}\right)$for each $i=$ $1, \ldots, n$. Then

$$
R\left(z_{0}\right)=\left(R^{1}\left(z_{0}\right), \ldots, R^{n}\left(z_{0}\right)\right)
$$

with

$$
R^{i}\left(z_{0}\right)=1, \quad i=1, \ldots, n
$$

is a necessary and sufficient condition that an equilibrium age distribution exists. The unique equilibrium age distribution $u_{0}=\left(u_{0}^{1}, \ldots, u_{0}^{n}\right)$ corresponding to $z_{0}=\left(z_{0}^{1}, \ldots, z_{0}^{n}\right)$ is given by

$$
u_{0}^{i}(a)=B_{0}^{i} \pi_{0}^{i}(a), \quad i=1, \ldots, n,
$$

where

$$
B_{0}^{i}=\frac{z_{0}^{i}}{\int_{0}^{\infty} \pi_{0}^{i}(a) d a} .
$$

Proof. The function (36) is the unique solution of $(31)_{1}$ with the initial condition $u_{0}^{i}(0)=B_{0}^{i}$. By $(31)_{2}$ we obtain the formula (37) for $B_{0}^{i}$. An easy computation shows that (35) is equivalent to $(31)_{3}$ for each $i=1, \ldots, n$.

We now turn to the problem of the equilibrium age distribution stability. We consider "perturbations" $\xi^{i}(a, t)$ and $p^{i}(t)$. Let us write

$$
\begin{aligned}
& u(a, t)= u_{0}(a)+\xi(a, t) \\
&=\left(u_{0}^{1}(a)+\xi^{1}(a, t), u_{0}^{2}(a)\right. \\
&\left.+\xi^{2}(a, t), \ldots, u_{0}^{n}(a)+\xi^{n}(a, t)\right) \\
& u^{i}(a, t)=u_{0}^{i}(a)+\xi^{i}(a, t),
\end{aligned}
$$


where $\xi^{i}(a, t)=\varphi^{i}(a, t)-u_{0}^{i}(a)$ for $t \in\left[-r^{i}, 0\right]$. And

$$
\begin{gathered}
z(t)=z_{0}+p(t) \\
=\left(z_{0}^{1}+p^{1}(t), z_{0}^{2}+p^{2}(t), \ldots, z_{0}^{n}+p^{n}(t)\right), \\
z_{t}=z_{0}+p_{t}=\left(z_{0}^{1}+p_{t}^{1}, z_{0}^{2}+p_{t}^{2}, \ldots, z_{0}^{n}+p_{t}^{n}\right), \\
z^{i}(t)=z_{0}^{i}+p^{i}(t), \\
z_{t}^{i}=z_{0}^{i}+p_{t}^{i},
\end{gathered}
$$

where $p_{t}^{i}(s)=p^{i}(t+s)$ for $s \in\left[-r^{i}, 0\right]$. Our goal is to formulate relations for $\xi^{i}$ and $p^{i}$ which guarantee that $u^{i}$ and $z_{0}^{i}$ obey the basic equations (1)-(4). From (1) we obtain

$$
D \xi^{i}(a, t)+\lambda_{0}^{i}(a) \xi^{i}(a, t)+\omega^{i}(a) p_{t}^{i}=x^{i}(a, t)
$$

for

$$
\begin{aligned}
x^{i}(a, t)= & -D_{z^{i}} \lambda^{i}\left(a, z_{0}\right) p_{t}^{i} \xi^{i}(a, t) \\
& -\Lambda^{i}\left(a, p_{t}\right)\left[B_{0}^{i} \pi_{0}^{i}(a)+\xi^{i}(a, t)\right], \\
& \lambda_{0}^{i}(a)=\lambda^{i}\left(a, z_{0}\right),
\end{aligned}
$$

where $\omega^{i}: \mathbb{R}_{+} \times C\left(\left[-r^{i}, 0\right]\right) \rightarrow \mathbb{R}$ is the functional

$$
\begin{gathered}
\omega^{i}(a) p_{t}^{i}=D_{z^{i}} \lambda^{i}\left(a, z_{0}\right) p_{t}^{i}\left(B_{0}^{i} \pi_{0}^{i}(a)\right) \\
\Lambda^{i}\left(a, p_{t}\right)=\lambda^{i}\left(a, z_{0}+p_{t}\right)-\lambda_{0}^{i}(a)-D_{z^{i}} \lambda^{i}\left(a, z_{0}\right) p_{t}^{i} .
\end{gathered}
$$

We conclude from (2) that

$$
\xi^{i}(0, t)=\int_{0}^{\infty} \int_{[-r, 0]} \beta_{0}^{i}(a) \xi^{i}\left(a, t+s^{i}\right) d s d a+\kappa^{i} p_{t}^{i}+\psi^{i}(t),
$$

where

$$
\begin{gathered}
\beta^{i}\left(a, z_{0}\right)=\beta_{0}^{i}(a), \\
\psi^{i}(t)=\int_{0}^{\infty} \int_{[-r, 0]} D_{z^{i}} \beta^{i}\left(a, z_{0}\right) p^{i}\left(t+s^{i}\right) \xi^{i}\left(a, t+s^{i}\right) d s d a \\
+\int_{0}^{\infty} \int_{[-r, 0]} \Omega^{i}(a, p(t+s)) \\
\times\left[B_{0}^{i} \pi_{0}^{i}(a)+\xi^{i}\left(a, t+s^{i}\right)\right] d s d a \\
\kappa^{i} p_{t}^{i}=B_{0}^{i} \int_{0}^{\infty} \int_{[-r, 0]} D_{z^{i}} \beta^{i}\left(a, z_{0}\right) p_{t}^{i} \pi_{0}^{i}(a) d s d a .
\end{gathered}
$$

Here $\kappa^{i}: C\left(\left[-r^{i}, 0\right]\right) \rightarrow \mathbb{R}$ is the functional. Moreover, we have

$$
\begin{aligned}
\Omega^{i}(a, p(t+s))= & \beta^{i}\left(a, z_{0}+p(t+s)\right) \\
& -\beta_{0}^{i}(a)-D_{z^{i}} \beta^{i}\left(a, z_{0}\right) p^{i}\left(t+s^{i}\right) .
\end{aligned}
$$

A trivial verification shows that (3) gives the condition for $p$ in the form

$$
p^{i}(t)=\int_{0}^{\infty} \xi^{i}(a, t) d a .
$$

We consider the system (42)-(48) with the initial condition

$$
\xi^{i}(a, 0)=\eta^{i}(a)
$$

where

$$
\eta^{i}(a)=\varphi^{i}(a, 0)-B_{0}^{i}
$$

We first express the solution of (42)-(48) in the form of the matrix equation. Let $\xi^{i}$ be the solution of (42) up to time $T$. Let $\left(a_{0}, t_{0}\right) \in \mathbb{R}_{+} \times[0, T], \overline{\xi^{i}}(h)=\xi^{i}\left(a_{0}+h, t_{0}+h\right), \overline{\lambda_{0}^{i}}(h)=$ $\lambda_{0}^{i}\left(a_{0}+h\right), \overline{\omega^{i}}(h)=\omega^{i}\left(a_{0}+h\right), \overline{p_{h}^{i}}=p_{t+h}^{i}$, and $\overline{x^{i}}(h)=x^{i}\left(a_{0}+\right.$ $\left.h, t_{0}+h\right)$. Then we can rewrite (42) as the equation

$$
\frac{d}{d h} \overline{\xi^{i}}(h)+\overline{\lambda_{0}^{i}}(h) \overline{\xi^{i}}(h)=\overline{x^{i}}(h)-\overline{\omega^{i}}(h) \overline{p_{h}^{i}}
$$

with the unique solution

$$
\begin{gathered}
\xi^{i}\left(a_{0}+h, t_{0}+h\right) \\
=\xi^{i}\left(a_{0}, t_{0}\right) e^{-\int_{0}^{h} \lambda_{0}^{i}\left(a_{0}+\tau\right) d \tau}+e^{-\int_{0}^{h} \lambda_{0}^{i}\left(a_{0}+\tau\right) d \tau} \\
\times \int_{0}^{h}\left[x^{i}\left(a_{0}+\tau, t_{0}+\tau\right)-\omega^{i}\left(a_{0}+\tau\right) p_{t_{0}+\tau}^{i}\right] \\
\times e^{\int_{0}^{\tau} \lambda_{0}^{i}\left(a_{0}+\alpha\right) d \alpha} d \tau .
\end{gathered}
$$

Substituting $\left(a_{0}, t_{0}\right)=(a-t, 0), h=t$ for $a \geqslant t$ and $\left(a_{0}, t_{0}\right)=$ $(0, t-a), h=a$ for $a<t$ yields the formulas

$$
\begin{aligned}
& \xi^{i}(a, t)=\eta^{i}(a-t) \tilde{\pi}^{i}(a-t, a) \\
& +\int_{0}^{t}\left[x^{i}(a-t+\tau, \tau)-\omega^{i}(a-t+\tau) p_{\tau}^{i}\right] \\
& \times \tilde{\pi}_{0}^{i}(a-t+\tau, a) d \tau \text { for } a \geqslant t, \\
& \xi^{i}(a, t)=b^{i}(t-a) \pi_{0}^{i}(a) \\
& +\int_{t-a}^{t}\left[x^{i}(\tau+a-t, \tau)-\omega^{i}(\tau+a-t) p_{\tau}^{i}\right] \\
& \times \tilde{\pi}_{0}^{i}(\tau+a-t, a) d \tau \text { for } a<t,
\end{aligned}
$$

where

$$
\begin{gathered}
\tilde{\pi}_{0}^{i}(a-t, a)=\frac{\pi_{0}^{i}(a)}{\pi_{0}^{i}(a-t)}, \\
b^{i}(t-a)=\xi^{i}(0, t-a) .
\end{gathered}
$$

Furthermore, (45) implies that

$$
p^{i}(t)=\int_{0}^{t} \xi^{i}(a, t) d a+\int_{t}^{\infty} \xi^{i}(a, t) d a .
$$


It follows that

$$
\begin{aligned}
& p^{i}(t)+\int_{0}^{t} \int_{0}^{\infty} \omega^{i}(a) p_{\tau}^{i} \tilde{\pi}_{0}^{i}(a, a+t-\tau) d a d \tau \\
& -\int_{0}^{t} b^{i}(\tau) \pi_{0}^{i}(t-\tau) d \tau \\
& \quad=\int_{0}^{t} \int_{t-\tau}^{\infty} x^{i}(\tau+a-t, \tau) \tilde{\pi}_{0}^{i}(\tau+a-t, a) d a d \tau \\
& \quad+\int_{0}^{\infty} \eta^{i}(\tau) \tilde{\pi}_{0}^{i}(\tau, \tau+t) d \tau .
\end{aligned}
$$

We conclude from (48) that

$$
\begin{aligned}
b^{i}(t)= & \int_{[-r, 0]} \int_{0}^{t+s^{i}} \beta_{0}^{i}(a) \xi^{i}\left(a, t+s^{i}\right) d a d s \\
& +\int_{[-r, 0]} \int_{t+s^{i}}^{\infty} \beta_{0}^{i}(a) \xi^{i}\left(a, t+s^{i}\right) d a d s \\
& +\kappa^{i}(t) p_{t}^{i}+\psi^{i}(t),
\end{aligned}
$$

and hence

$$
\begin{aligned}
& -\kappa^{i} p_{t}^{i}+b^{i}(t) \\
& +\int_{0}^{t} \int_{-[r, 0]} \int_{0}^{\infty} \beta_{0}^{i}\left(a-\tau+t+s^{i}\right) \omega^{i}(a) p_{\tau}^{i} \\
& \times \tilde{\pi}_{0}^{i}\left(a, a-\tau+t+s^{i}\right) d a d s d \tau \\
& -\int_{0}^{t} \int_{[-r, 0]} \beta_{0}^{i}\left(t+s^{i}-\tau\right) b^{i}(\tau) \pi_{0}^{i}\left(t+s^{i}-\tau\right) d s d \tau
\end{aligned}
$$

$$
\begin{gathered}
\int_{[-r, 0]} \int_{0}^{t+s^{i}} \int_{t+s^{i}-\tau}^{\infty} \beta_{0}^{i}(a) x^{i}\left(\tau+a-t-s^{i}, \tau\right) \\
\times \tilde{\pi}_{0}^{i}\left(\tau+a-t-s^{i}, a\right) d a d \tau d s \\
+\int_{[-r, 0]} \int_{0}^{\infty} \beta_{0}^{i}\left(\tau+t+s^{i}\right) \eta^{i}(\tau) \\
\times \tilde{\pi}_{0}^{i}\left(\tau, \tau+t+s^{i}\right) d \tau d s+\psi^{i}(t) .
\end{gathered}
$$

Conditions (57) and (59) imply that

$$
\mathbf{y}(t)=\left[\begin{array}{c}
\mathbf{y}^{1}(t) \\
\vdots \\
\mathbf{y}^{n}(t)
\end{array}\right]
$$

where

$$
\begin{gathered}
\mathbf{y}^{i}(t)=\left[\begin{array}{l}
p^{i}(t) \\
b^{i}(t)
\end{array}\right], \\
\mathbf{y}_{t}^{i}(s)=\mathbf{y}^{i}(t+s) \quad \text { for } s \in\left[-r^{i}, 0\right], i=1,2, \ldots, n,
\end{gathered}
$$

satisfy the matrix equation

$$
\mathbf{A y}_{t}+\int_{0}^{t} \mathbf{K}(t-\tau) \mathbf{y}_{\tau} d \tau=\mathbf{f}(t)
$$

where $\mathbf{A}$ and $\mathbf{K}$ are block diagonal matrices. We have

$$
\mathbf{A}=\left[\begin{array}{cccc}
\mathbf{A}^{1} & \mathbf{O} & \ldots & \mathbf{O} \\
\mathbf{O} & \mathbf{A}^{2} & \ldots & \mathbf{O} \\
\vdots & \vdots & \ddots & \vdots \\
\mathbf{O} & \mathbf{O} & \ldots & \mathbf{A}^{n}
\end{array}\right], \quad \mathbf{K}=\left[\begin{array}{cccc}
\mathbf{K}^{1} & \mathbf{O} & \ldots & \mathbf{O} \\
\mathbf{O} & \mathbf{K}^{2} & \ldots & \mathbf{O} \\
\vdots & \vdots & \ddots & \vdots \\
\mathbf{O} & \mathbf{O} & \ldots & \mathbf{K}^{n}
\end{array}\right]
$$

for zero matrix $\mathbf{O}, \mathbf{A}^{i}=\left[\begin{array}{cc}1 & 0 \\ -\kappa^{i} & 1\end{array}\right], \mathbf{A}^{i} \mathbf{y}_{t}=\left[\begin{array}{cc}p_{t}^{i}(0) & 0 \\ -\kappa^{i} p_{t}^{i} & b_{t}^{i}(0)\end{array}\right]=$ $\left[\begin{array}{cc}p^{i}(t) & 0 \\ -\kappa^{i} p_{t}^{i} b^{i}(t)\end{array}\right]$ and

$$
\mathbf{K}^{i}(t)=\left[\begin{array}{cc}
\int_{0}^{\infty} \tilde{\pi}_{0}^{i}(a, a+t) \omega^{i}(a) d a & -\pi_{0}^{i}(t) \\
\int_{-r^{i}}^{0} \int_{0}^{\infty} \beta_{0}^{i}(a+t+s) \tilde{\pi}_{0}^{i}(a, a+t+s) \omega^{i}(a) d a d s & -\int_{-r^{i}}^{0} \beta_{0}^{i}(t+s) \pi_{0}^{i}(t+s) d s
\end{array}\right] .
$$

Since $\omega(a)$ is the functional so in the formula of $\mathbf{K}(t)$ we consider $\omega$ as the function with functional values. Furthermore, in the notation (63) looks like the ordinary matrix equation, but in fact it is the operator one. Moreover,

$$
\mathbf{f}(t)=\left[\begin{array}{c}
\mathbf{f}^{1}(t) \\
\vdots \\
\mathbf{f}^{n}(t)
\end{array}\right]
$$

for

$$
\begin{aligned}
\mathbf{f}^{i}(t) & =\left[\begin{array}{l}
f_{1}^{i}(t) \\
f_{2}^{i}(t)
\end{array}\right]-\mathbf{A}^{i} \mathbf{y}_{1 t}^{i}-\int_{0}^{t} \mathbf{K}^{i}(t-\tau) \mathbf{y}_{1 \tau}^{i} d \tau \\
f_{1}^{i}(t)= & \int_{0}^{t} \int_{t-\tau}^{\infty} x^{i}(\tau+a-t, \tau) \tilde{\pi}_{0}^{i}(\tau+a-t, a) d a d \tau \\
& +\int_{0}^{\infty} \eta^{i}(\tau) \tilde{\pi}_{0}^{i}(\tau, \tau+t) d \tau,
\end{aligned}
$$




$$
\begin{array}{r}
f_{2}^{i}(t)=\int_{[-r, 0]} \int_{0}^{t+s^{i}} \int_{t+s^{i}-\tau}^{\infty} \beta_{0}^{i}(a) x^{i}\left(\tau+a-t-s^{i}, \tau\right) \\
\times \tilde{\pi}_{0}^{i}\left(\tau+a-t-s^{i}, a\right) d a d \tau d s \\
+\int_{[-r, 0]} \int_{0}^{\infty} \beta_{0}^{i}\left(\tau+t+s^{i}\right) \eta^{i}(\tau) \\
\times \tilde{\pi}_{0}^{i}\left(\tau, \tau+t+s^{i}\right) d \tau d s+\psi^{i}(t)
\end{array}
$$

and $\mathbf{y}_{1}^{i}(t)=\left.\left[\begin{array}{c}p^{i}(t) \\ 0\end{array}\right]\right|_{\left[-r^{i}, 0\right]}, \mathbf{y}_{1 t}^{i}(s)=\mathbf{y}_{1}^{i}(t+s)$ for $s \in\left[-r^{i}, 0\right]$.

We can notice that the last two elements of the expression defining $\mathbf{f}^{i}(t)$ equal zero for $t>r$.

We return to the previous deliberation in the next theorem. We take some additional assumptions.

$\left(H_{10}\right)$ The Fréchet derivatives $D_{z^{i}} \lambda^{i}\left(a, z_{0}\right)$ and the derivation $D_{z^{i}} \beta^{i}\left(a, z_{0}\right)$ for $i=1,2 \ldots, n$ as functions of the variable $a$ belong to $L^{\infty}\left(\mathbb{R}_{+}\right)$.

$\left(H_{11}\right)\left(\Lambda^{i}(a, \rho) /\|\rho\|_{0}\right)$ and $\left(\Omega^{i}(a, \rho) /\|\rho\|_{0}\right)$ tend to zero as $\|\rho\|_{0} \rightarrow 0$ uniformly for $a \rightarrow 0$; here $\|\cdot\|_{0}$ denotes the supremum norm in the Banach space $C\left(\left[-r^{i}, 0\right]\right)$.

$\left(H_{12}\right) \lambda_{*}=\inf _{a \geqslant 0}\left\{\lambda^{i}\left(a, z_{0}\right), i=1,2, \ldots, n\right\}>0$.

Denote $e_{\theta}:(-\infty, 0] \rightarrow \mathbb{R}$ by the formula $e_{\theta}(s)=e^{\theta s}$. The exponential asymptotical stability of the model is established by the following theorem.

Theorem 4. Let $\tilde{\pi}_{1}^{i}(a, a+t)=\int_{[-r, 0]} \beta_{0}^{i}\left(a+t+s^{i}\right) \tilde{\pi}_{0}^{i}(a, a+t+$ $\left.s^{i}\right) d s$, and let $\pi_{1}^{i}(t)=\int_{[-r, 0]} \beta_{0}^{i}\left(t+s^{i}\right) \pi_{0}^{i}\left(t+s^{i}\right) d s$. Let us assume that there exists some $\bar{\mu}>0$ that the equation

$$
\begin{aligned}
1= & \int_{0}^{\infty} e^{-t \gamma} \pi_{1}^{i}(t) d t \\
& -\int_{0}^{\infty} e^{-t \gamma} \int_{0}^{\infty} \tilde{\pi}_{0}^{i}(a, a+t) \omega^{i}(a)\left(e_{\gamma}\right) d a d t \\
& \cdot\left(1-\int_{0}^{\infty} e^{-t \gamma} \pi_{1}^{i}(t) d t\right) \\
& -\int_{0}^{\infty} e^{-t \gamma} \pi_{0}^{i}(t) d t \\
& \cdot\left(\int_{0}^{\infty} e^{-t \gamma} \int_{0}^{\infty} \tilde{\pi}_{1}^{i}(a, a+t) \omega^{i}(a)\left(e_{\gamma}\right) d a d t-\kappa^{i}\left(e_{\gamma}\right)\right)
\end{aligned}
$$

has no solution $\gamma$ with $\operatorname{Re}(\gamma) \geqslant-\bar{\mu}$. Then there exist real numbers $\delta>0$ and $\mu>0$ such that for any initial data $\varphi$ with $\left\|\varphi-u_{0}\right\|_{L^{1}}<\delta$, the corresponding solution of the population problem (1)-(4), if it exists for $t>0$, satisfies

$$
\begin{gathered}
\left\|z_{t}-z_{0}\right\|=O\left(e^{-\mu t}\right), \\
\left\|u(a, t)-u_{0}(a)\right\|=O\left(e^{-\mu t}\right) \text { for each } a
\end{gathered}
$$

Proof. Let $u$ be the solution of the population problem (1)(4) for $t>0$. In the proof we will use the properties of the generalized Laplace transform. Let $\varrho:[0, \infty) \rightarrow C\left(\left[-r^{i}, 0\right]\right)^{*}$ be the function with measure value. Define

$$
\widehat{\varrho}(\theta)=\int_{0}^{\infty} \varrho(t)\left(e_{\theta}\right) e^{-\theta t} d t .
$$

If we define the convolution by the formula

$$
\left(\varrho * v_{\tau}\right)(t)=\int_{0}^{t} \varrho(t-\tau) v(\tau+\cdot) d \tau,
$$

where $v(t)=0$ for $t<0$, then we have the equality analogous to the property of classical Laplace transform, that is,

$$
\widehat{\varrho * v_{\tau}}(\theta)=\widehat{\varrho}(\theta) \widehat{v}(\theta) .
$$

Using generalized Laplace transform to (63) we get

$$
\widehat{\mathbf{A}}(\theta) \widehat{\mathbf{y}}(\theta)+\widehat{\mathbf{K}}(\theta) \widehat{\mathbf{y}}(\theta)=\widehat{\mathbf{f}}(\theta),
$$

where

$$
\begin{aligned}
\widehat{\mathbf{A}}(\theta)= & {\left[\begin{array}{cccc}
\widehat{\mathbf{A}}^{1}(\theta) & \mathbf{O} & \cdots & \mathbf{O} \\
\mathbf{O} & \widehat{\mathbf{A}}^{2}(\theta) & \cdots & \mathbf{O} \\
\vdots & \vdots & \ddots & \vdots \\
\mathbf{O} & \mathbf{O} & \cdots & \widehat{\mathbf{A}}^{n}(\theta)
\end{array}\right], } \\
& \widehat{\mathbf{A}}^{i}(\theta)=\left[\begin{array}{ccc}
1 & 0 \\
-\kappa^{i}\left(e_{\theta}\right) & 1
\end{array}\right]
\end{aligned}
$$$$
\widehat{\mathbf{K}}(\theta)=\left[\begin{array}{cccc}
\widehat{\mathbf{K}}^{1}(\theta) & \mathbf{O} & \cdots & \mathbf{O} \\
\mathbf{O} & \widehat{\mathbf{K}}^{2}(\theta) & \cdots & \mathbf{O} \\
\vdots & \vdots & \ddots & \vdots \\
\mathbf{O} & \mathbf{O} & \cdots & \widehat{\mathbf{K}}^{n}(\theta)
\end{array}\right]
$$

$\widehat{\mathbf{K}}^{i}(\theta)$

$$
=\left[\begin{array}{l}
\int_{0}^{\infty} e^{-t \theta} \int_{0}^{\infty} \tilde{\pi}_{0}^{i}(a, a+t) \omega^{i}(a)\left(e_{\theta}\right) d a d t-\hat{\pi}_{0}^{i}(\theta) \\
\int_{0}^{\infty} e^{-t \theta} \int_{0}^{\infty} \tilde{\pi}_{1}^{i}(a, a+t) \omega^{i}(a)\left(e_{\theta}\right) d a d t-\hat{\pi}_{1}^{i}(\theta)
\end{array}\right]
$$

for $\tilde{\pi}_{1}^{i}(a, a+t)=\int_{[-r, 0]} \beta_{0}^{i}\left(a+t+s^{i}\right) \tilde{\pi}_{0}^{i}\left(a, a+t+s^{i}\right) d s$ and $\pi_{1}^{i}(t)=\int_{[-r, 0]} \beta_{0}^{i}\left(t+s^{i}\right) \pi_{0}^{i}\left(t+s^{i}\right) d s$. From the equality (68) we conclude that the matrix $\widehat{\mathbf{A}}+\widehat{\mathbf{K}}$ has an analytic inverse for $\operatorname{Re}(\theta) \geqslant-\bar{\mu}$. Moreover, we have

$$
\begin{gathered}
\widehat{\mathbf{A}}^{-1}(\theta)=\left[\begin{array}{cccc}
\left(\widehat{\mathbf{A}}^{1}\right)^{-1}(\theta) & \mathbf{O} & \cdots & \mathbf{O} \\
\mathbf{O} & \left(\widehat{\mathbf{A}}^{2}\right)^{-1}(\theta) & \cdots & \mathbf{O} \\
\vdots & \vdots & \ddots & \vdots \\
\mathbf{O} & \mathbf{O} & \cdots & \left(\widehat{\mathbf{A}}^{n}\right)^{-1}(\theta)
\end{array}\right] \\
\left(\widehat{\mathbf{A}}^{i}\right)^{-1}(\theta)=\left[\begin{array}{cc}
1 & 0 \\
\kappa^{i}\left(e_{\theta}\right) & 1
\end{array}\right]
\end{gathered}
$$

so $\widehat{\mathbf{A}}$ is analytic for $\theta \in \mathbb{C}$. 
The solution of the matrix equation (63) exists and is given by

$$
\mathbf{y}(t)=\mathbf{A}^{-1} \mathbf{f}_{t}+\int_{0}^{t} \mathbf{J}(t-\tau) \mathbf{f}_{\tau} d \tau,
$$

where $\mathbf{f}_{\tau}(s)=\left[\begin{array}{c}\mathbf{f}^{1}(\tau+s) \\ \vdots \\ \mathbf{f}^{n}(\tau+s)\end{array}\right], \mathbf{f}_{\tau}^{i}(s)=\mathbf{f}^{i}(\tau+s)$ for $s \in\left[-r^{i}, 0\right]$ and

$$
\mathbf{J}(t)=\frac{1}{2 \pi} e^{-\bar{\mu} t} \int_{-\infty}^{\infty} e^{i \zeta t} \mathbf{J}(-\bar{\mu}+i \zeta) d \zeta .
$$

$\widehat{\mathbf{J}}=(\widehat{\mathbf{A}}(\theta)+\widehat{\mathbf{K}}(\theta))^{-1}-\widehat{\mathbf{A}}^{-1}(\theta)$ is the Laplace transform of the function $\mathbf{J}$ and

$$
|\mathbf{J}(t)| \leqslant C_{1} e^{-\bar{\mu} t},
$$

where $|\mathbf{J}|$ denotes the sum of moduli of the elements of the matrix J. Here and in the whole proof $C_{1}, C_{2}, C_{3}, \ldots$ denote positive constants.

By $\left(H_{12}\right),(32)$, and (54)

$$
\pi_{0}^{i}(a) \leqslant e^{-\lambda_{*} a}, \quad \tilde{\pi}_{0}^{i}\left(a^{\prime}, a\right) \leqslant e^{-\lambda_{*}\left(a-a^{\prime}\right)} \quad\left(a^{\prime} \leqslant a\right) .
$$

For $\lambda_{*}>\bar{\mu}$ and $\left\|\beta_{0}\right\|_{L^{\infty}}<\infty$ we can estimate that

$$
\begin{gathered}
\|\mathbf{f}(t)\| \leqslant C_{2}\left\{\int_{0}^{t} e^{-\lambda_{*}(t-\tau)}\|x(\cdot, \tau)\|_{L^{1}} d \tau\right. \\
\left.+\|\eta\|_{L^{1}} e^{-\lambda_{*} t}+\|\psi(t)\|_{L^{1}}\right\}, \\
\|\mathbf{y}(t)\| \leqslant C_{3}\left\{\|\eta\|_{L^{1}} e^{-\bar{\mu} t}+\|\psi(t)\|_{L^{1}}\right. \\
\left.+\int_{0}^{t} e^{-\bar{\mu}(t-\tau)}\left[\|\psi(\tau)\|_{L^{1}}+\|x(\cdot, \tau)\|_{L^{1}}\right] d \tau\right\},
\end{gathered}
$$

where $\|\mathbf{y}(t)\|=\left(\sum_{i=1}^{n}\left(p^{i}\right)^{2}+\left(b^{i}\right)^{2}\right)^{1 / 2}$

$$
\begin{aligned}
\|\xi(\cdot, t)\|_{L^{1}} \leqslant & \int_{0}^{t} e^{-\lambda_{*} \tau}\|b(t-\tau)\| d \tau+\|\eta\|_{L^{1}} e^{-\lambda_{*} t} \\
& +\int_{0}^{t} e^{-\lambda_{*}(t-\tau)}\left(\|x(\cdot, \tau)\|_{L^{1}}+\|\omega\|_{L^{1}}\left\|p_{\tau}\right\|_{0}\right) d \tau \\
\leqslant & C_{4}\left\{\|\eta\|_{L^{1}} e^{-\bar{\mu} t}\right. \\
& \quad \int_{0}^{t} e^{-\bar{\mu}(t-\tau)} \\
& \left.\times\left[\|\psi(\tau)\|_{L^{1}}+\|x(\cdot, \tau)\|_{L^{1}}\right] d \tau\right\}
\end{aligned}
$$

Let $\varepsilon>0$. By $\left(H_{11}\right)$ there exists $\widetilde{\delta}=\widetilde{\delta}(\varepsilon)$ such that

$$
\left\|\Lambda\left(a, p_{t}\right)\right\| \leqslant \varepsilon\left\|p_{t}\right\|_{0}, \quad\left\|\Omega\left(a, p_{t}\right)\right\| \leqslant \varepsilon\left\|p_{t}\right\|_{0}
$$

for $\left\|p_{t}\right\|_{0}<\tilde{\delta}$. By the previous inequalities and the fact that $\left\|D_{z} \lambda\left(a, z_{0}\right)\right\|_{L^{\infty}},\left\|D_{z} \beta\left(a, z_{0}\right)\right\|_{L^{\infty}}$, and $\left\|u_{0}\right\|_{L^{1}}$ are finite, we can estimate that

$$
\|x(\cdot, t)\|_{L^{1}},\|\psi(t)\|_{L^{1}} \leqslant C_{5}\left\{\left\|p_{t}\right\|_{0} \cdot\|\xi(\cdot, t)\|_{L^{1}}+\varepsilon\left\|p_{t}\right\|_{0}\right\} .
$$

We require that $\varepsilon<1$ and $\widetilde{\delta}<\varepsilon$. Hence $\left\|p_{t}\right\|_{0}<\varepsilon$. According to the previous remarks, we have

$$
\begin{gathered}
\|x(\cdot, t)\|_{L^{1}},\|\psi(t)\|_{L^{1}} \leqslant C_{5} \varepsilon \sigma(t), \\
\sigma(t)=\|\xi(\cdot, t)\|_{L^{1}}+\left\|p_{t}\right\|_{0^{0}} .
\end{gathered}
$$

Thus (82)-(86) imply that

$$
\sigma(t) \leqslant M\left\{\|\eta\|_{L^{1}} e^{-\bar{\mu} t}+2 \varepsilon \int_{0}^{t} e^{-\bar{\mu}(t-\tau)} \sigma(\tau) d \tau\right\},
$$

where $M>0$. Gronwall's inequality yields

$$
\sigma(t) \leqslant M\|\eta\|_{L^{1}} e^{(-\bar{\mu}+2 M \varepsilon) t}
$$

Let us choose $\delta$ and $\varepsilon>0$ such that $\mu=\bar{\mu}-2 M \varepsilon>0$ and $\delta<$ $\min (\widetilde{\delta}(\varepsilon), \widetilde{\delta}(\varepsilon) / M)$. Assume that $\left\|\varphi-u_{0}\right\|_{L^{1}}=\|\eta\|_{L^{1}}<\delta$. In that case, from what has already been proved, it follows that $\|x(\cdot, t)\|_{L^{1}},\|\psi(t)\|_{L^{1}}, \sigma(t)$ and $\|b(t)\|$ are $O\left(e^{-\mu t}\right)$. Therefore (41), (86) $)_{2}$ imply (69) and (53), (80), and (39) imply (70).

Example 5. Let $n=2$. Let us consider

$$
\begin{gathered}
\lambda^{i}(a, z)=\lambda^{i}\left(a, z_{1}^{1}, z_{2}^{1}, z_{1}^{2}, z_{2}^{2}\right), \\
\beta^{i}(a, z)=\beta^{i}\left(a, z^{1}, z^{2}\right),
\end{gathered}
$$

where

$$
\begin{gathered}
\lambda^{i}(a, z)=\lambda^{i}\left(z^{1}(0), z^{1}\left(-r^{1}\right), z^{2}(0), z^{2}\left(-r^{2}\right)\right), \\
\beta^{i}(a, z)=\beta^{i}\left(z^{1}, z^{2}\right) e^{-\alpha^{i} a}
\end{gathered}
$$

with $\lambda^{i}>0, \beta^{i}>0, r^{i}>0, \alpha^{i}>0$ for $i=1,2$. Let us define

$$
\begin{gathered}
\lambda_{0}^{i}=\lambda^{i}\left(z_{0}^{1}, z_{0}^{1}, z_{0}^{2}, z_{0}^{2}\right), \\
\beta_{0}^{i}=\beta^{i}\left(z_{0}^{1}, z_{0}^{2}\right) e^{-\alpha^{i} a}
\end{gathered}
$$

for an arbitrary $z_{0}=\left(z_{0}^{1}, z_{0}^{2}\right) \in \mathbb{R}^{2}$. From (32) and (33) we conclude that

$$
\begin{gathered}
\pi_{0}^{i}(a)=e^{-a \lambda_{0}^{i}}, \\
R^{i}\left(z_{0}\right)=\frac{r^{1} r^{2} \beta_{0}^{i}}{\alpha^{i}+\lambda_{0}^{i}} .
\end{gathered}
$$

By Theorem 3 there exists an equilibrium age distribution $u_{0}^{i}(a)=z_{0}^{i} \lambda_{0}^{i} e^{-\lambda_{0}^{i} a}$ where $B_{0}=z_{0}^{i} \lambda_{0}^{i}$ if and only if $r^{1} r^{2} \beta_{0}^{i} /\left(\alpha^{i}+\right.$ $\left.\lambda_{0}^{i}\right)=1$. To investigate the stability of the equilibrium age 
distribution we consider (68). Let $z_{0}=\left(z_{0}^{1}, z_{0}^{2}\right)$ be the solution of the system of the equations

$$
R^{i}\left(z_{0}\right)=1, \quad i=1,2 .
$$

First, let us notice that

$$
\omega^{i}\left(e_{\gamma}\right)=\left(\frac{\partial \lambda^{i}}{\partial z_{1}^{i}}+\frac{\partial \lambda^{i}}{\partial z_{2}^{i}} e^{-\gamma r^{i}}\right) \lambda_{0}^{i} z_{0}^{i} e^{-a \lambda_{0}^{i}}
$$

with the derivatives in the point $z_{0}$. It is easy to notice that $\left|\partial \lambda^{i} / \partial z_{1}^{i}+\left(\partial \lambda^{i} / \partial z_{2}^{i}\right) e^{-\gamma r^{i}}\right|$ is bounded on half-plane $\operatorname{Re}(\gamma)>\mu$ for every real $\mu$ (also negative). Analogously

$$
\kappa^{i}\left(e_{\gamma}\right)=\frac{\lambda_{0}^{i}}{\beta_{0}^{i}} \cdot \frac{z_{0}^{i}}{r^{i}} \cdot \frac{\partial \beta^{i}}{\partial z^{i}}\left(z_{0}\right) \int_{-r^{i}}^{0} e^{\gamma s} d s .
$$

It is also possible to prove that for every real $\mu$ the function $\left|\kappa^{i}\left(e_{\gamma}\right)\right|$ is bounded on half-plane $\operatorname{Re}(\gamma)>\mu$. Consider the right-hand side of (68). All components can be presented in the form

$$
\frac{A}{B+\gamma}
$$

where $A$ is bounded and $B \geqslant \max \left\{\alpha^{i}, \lambda_{0}^{i}\right\}$. Let $\mu<B$, and let $\operatorname{Re}(\gamma)>-\mu$ :

$$
\begin{aligned}
\left|\frac{A}{B+\gamma}\right| & =\left|\frac{A}{B-\mu+\mu+\gamma}\right| \leq\left|\frac{A}{B-\mu+\mu+\operatorname{Re}(\gamma)}\right| \\
& \leq\left|\frac{A}{2 \sqrt{B-\mu} \sqrt{\mu+\operatorname{Re}(\gamma)}}\right| .
\end{aligned}
$$

Fix $\sigma>0$. For $B$ sufficiently large there exist some $\mu$ and $\bar{\mu}<\mu$ that

$$
\left|\frac{A}{2 \sqrt{B-\mu} \sqrt{\mu-\bar{\mu}}}\right|<\sigma .
$$

For $\operatorname{Re}(\gamma)>-\bar{\mu}$ we have

$$
\left|\frac{A}{B+\gamma}\right| \leqslant\left|\frac{A}{2 \sqrt{B-\mu} \sqrt{\mu+\operatorname{Re}(\gamma)}}\right| \leqslant\left|\frac{A}{2 \sqrt{B-\mu} \sqrt{\mu-\bar{\mu}}}\right|<\sigma .
$$

In consequence for $\alpha^{i}$ and $\lambda_{0}^{i}$ sufficiently large, we can find that $\bar{\mu}>0$ such that the modulus of the right-hand side of (68) is less than 1 for every $\gamma$, for which $\operatorname{Re}(\gamma)>-\bar{\mu}$.

\section{Acknowledgment}

The first author acknowledges the support from Bialystok University of Technology (Grant no. S/WI/2/2011).

\section{References}

[1] A. Hastings, "Interacting age structured populations," in Mathematical Ecology, vol. 17 of Biomathematics, pp. 287-294, Springer, Berlin, Germany, 1986.

[2] K. R. Fister and S. Lenhart, "Optimal harvesting in an agestructured predator-prey model," Applied Mathematics and Optimization, vol. 54, no. 1, pp. 1-15, 2006.

[3] Z. He and H. Wang, "Control problems of an age-dependent predator-prey system," Applied Mathematics, vol. 24, no. 3, pp. 253-262, 2009.

[4] D. S. Levine, "Bifurcating periodic solutions for a class of age-structured predator-prey systems," Bulletin of Mathematical Biology, vol. 45, no. 6, pp. 901-915, 1983.

[5] D. J. Wollkind, A. Hastings, and J. A. Logan, "Functionalresponse, numerical response, and stability in arthropod predator-prey ecosystems involving age structure," Researches on Population Ecology, vol. 22, pp. 323-338, 1980.

[6] M. E. Gurtin and R. C. MacCamy, "Non-linear age-dependent population dynamics," Archive for Rational Mechanics and Analysis, vol. 54, pp. 281-300, 1974.

[7] D. Breda, M. Iannelli, S. Maset, and R. Vermiglio, "Stability analysis of the Gurtin-MacCamy model," SIAM Journal on Numerical Analysis, vol. 46, no. 2, pp. 980-995, 2008.

[8] J. M. Cushing, "The dynamics of hierarchical age-structured populations," Journal of Mathematical Biology, vol. 32, no. 7, pp. 705-729, 1994.

[9] A. L. Dawidowicz and A. Poskrobko, "Age-dependent singlespecies population dynamics with delayed argument," Mathematical Methods in the Applied Sciences, vol. 33, no. 9, pp. 1122$1135,2010$.

[10] A. L. Dawidowicz and A. Poskrobko, "On the age-dependent population dynamics with delayed dependence of the structure," Nonlinear Analysis. Theory, Methods \& Applications, vol. 71, no. 12, pp. e2657-e2664, 2009.

[11] G. Di Blasio, "Nonlinear age-dependent population growth with history-dependent birth rate," Mathematical Biosciences, vol. 46, no. 3-4, pp. 279-291, 1979.

[12] V. G. Matsenko, "A nonlinear model of the dynamics of the age structure of populations," Nelīnīnī Kolivannya, vol. 6, no. 3, pp. 357-367, 2003.

[13] S. Piazzera, "An age-dependent population equation with delayed birth process," Mathematical Methods in the Applied Sciences, vol. 27, no. 4, pp. 427-439, 2004.

[14] K. E. Swick, "A nonlinear age-dependent model of single species population dynamics," SIAM Journal on Applied Mathematics, vol. 32, no. 2, pp. 484-498, 1977.

[15] K. E. Swick, "Periodic solutions of a nonlinear age-dependent model of single species population dynamics," SIAM Journal on Mathematical Analysis, vol. 11, no. 5, pp. 901-910, 1980.

[16] Z. G. Bao and W. L. Chan, "A semigroup approach to agedependent population dynamics with time delay," Communications in Partial Differential Equations, vol. 14, no. 6, pp. 809-832, 1989.

[17] O. Arino, E. Sánchez, and G. F. Webb, "Necessary and sufficient conditions for asynchronous exponential growth in age structured cell populations with quiescence," Journal of Mathematical Analysis and Applications, vol. 215, no. 2, pp. 499-513, 1997.

[18] O. Arino, E. Sánchez, and G. F. Webb, "Polynomial growth dynamics of telomere loss in a heterogeneous cell population," Dynamics of Continuous, Discrete and Impulsive Systems, vol. 3, no. 3, pp. 263-282, 1997. 
[19] F. Billy, J. Clairambault, O. Fercoq et al., "Synchronisation and control of proliferation in cycling cell population models with age structure," Mathematics and Computers in Simulation, 2012.

[20] H. Inaba, "Strong ergodicity for perturbed dual semigroups and application to age-dependent population dynamics," Journal of Mathematical Analysis and Applications, vol. 165, no. 1, pp. 102132, 1992.

[21] I. Roeder, M. Herberg, and M. Horn, "An "age"-structured model of hematopoietic stem cell organization with application to chronic myeloid leukemia," Bulletin of Mathematical Biology, vol. 71, no. 3, pp. 602-626, 2009.

[22] R. Rundnicki and M. C. Mackey, "Asymptotic similarity and Malthusian growth in autonomous and nonautonomous populations," Journal of Mathematical Analysis and Applications, vol. 187, no. 2, pp. 548-566, 1994.

[23] J. von Foerster, Some Remarks on Changing Populations, the Kinetics of Cell Proliferation, Grune \& Stratton, New York, NY, USA, 1959. 


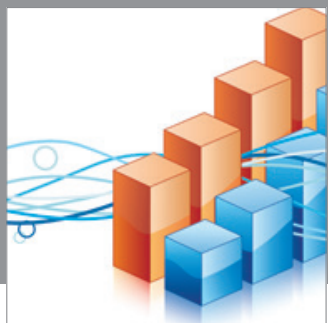

Advances in

Operations Research

mansans

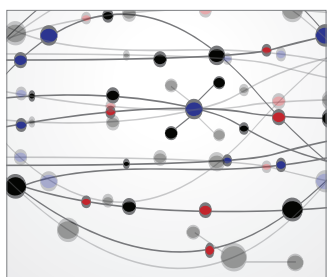

The Scientific World Journal
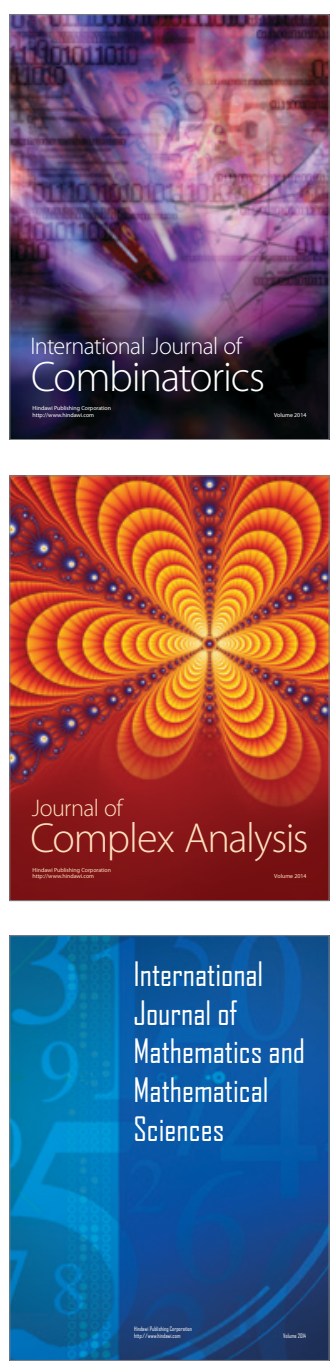
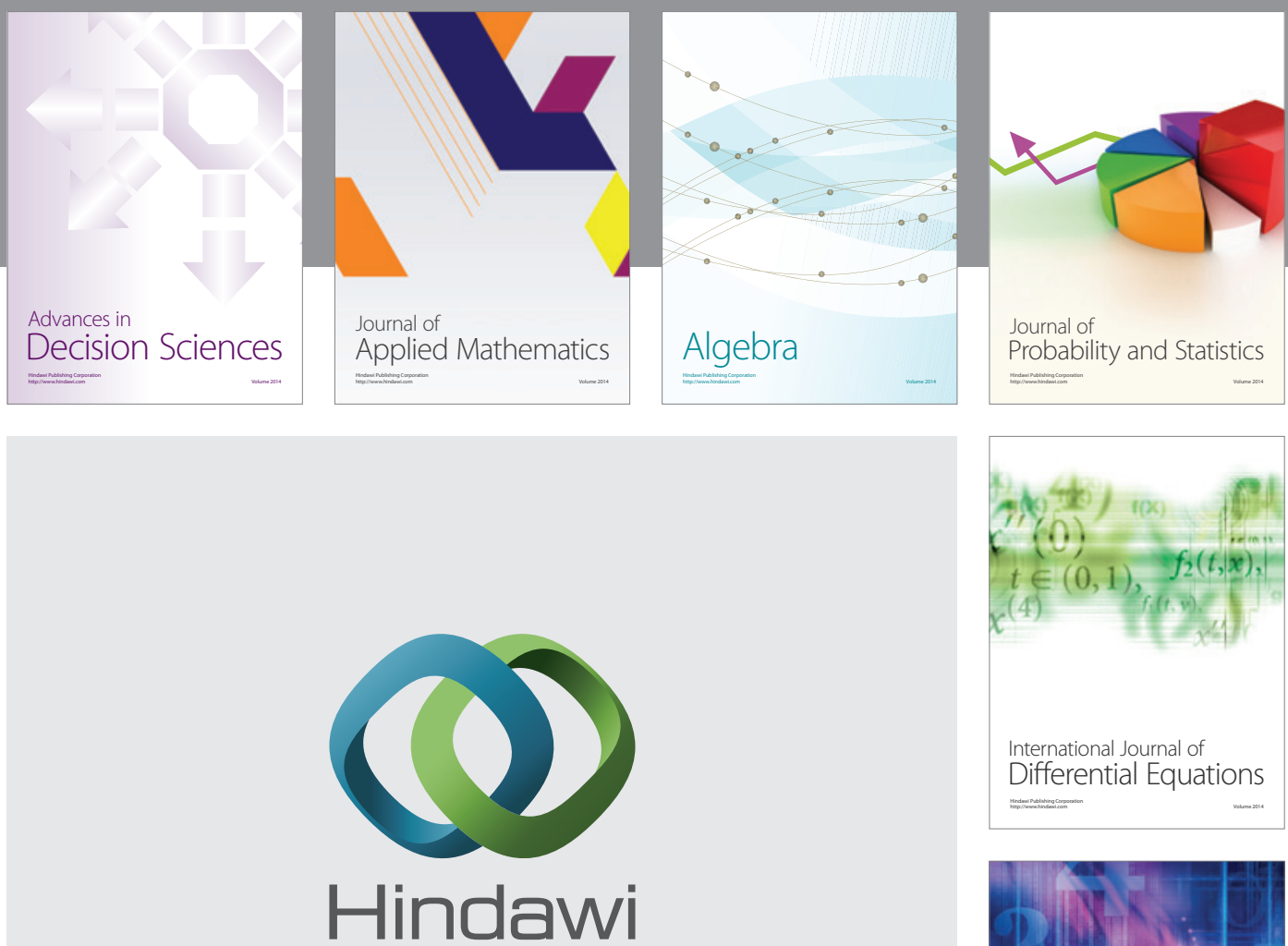

Submit your manuscripts at http://www.hindawi.com
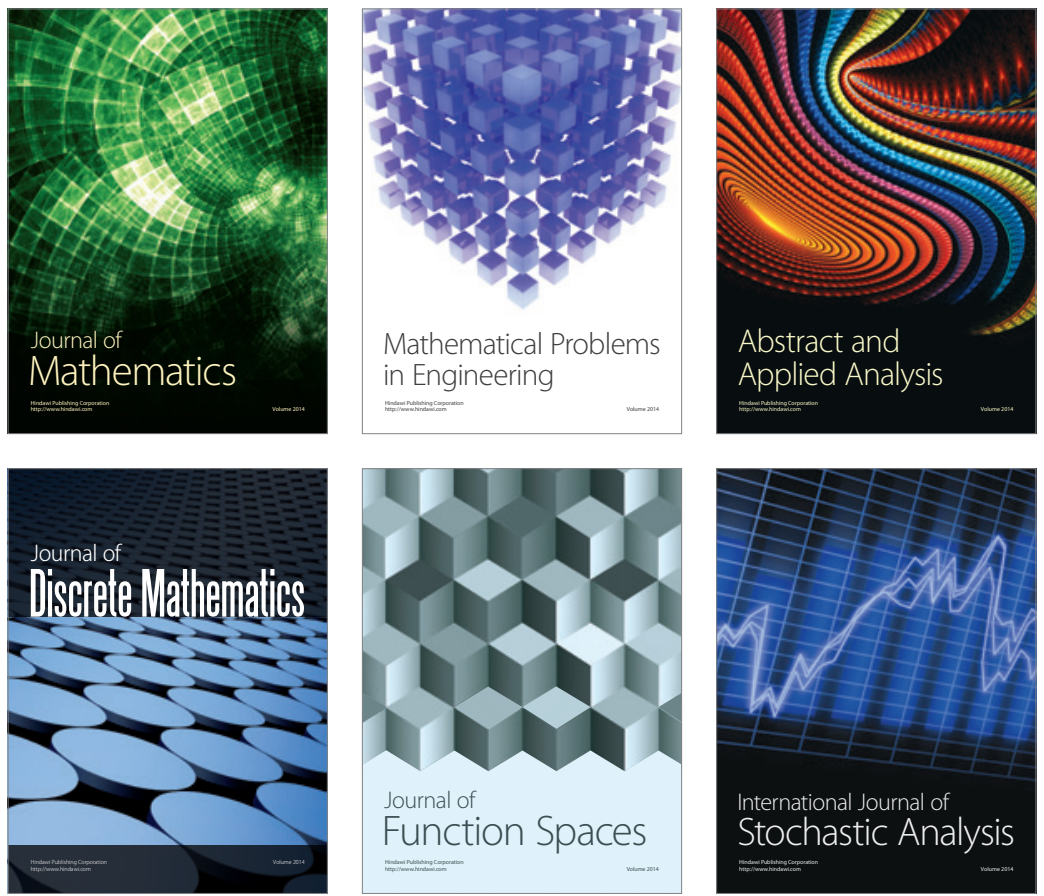

Journal of

Function Spaces

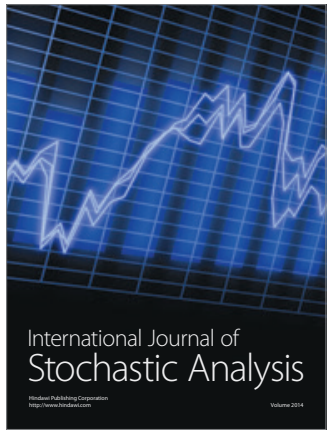

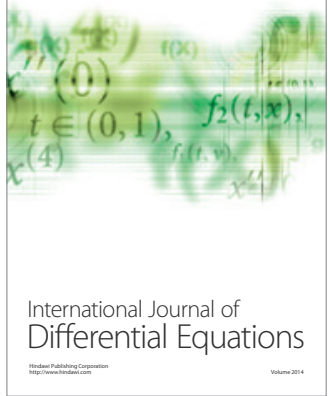
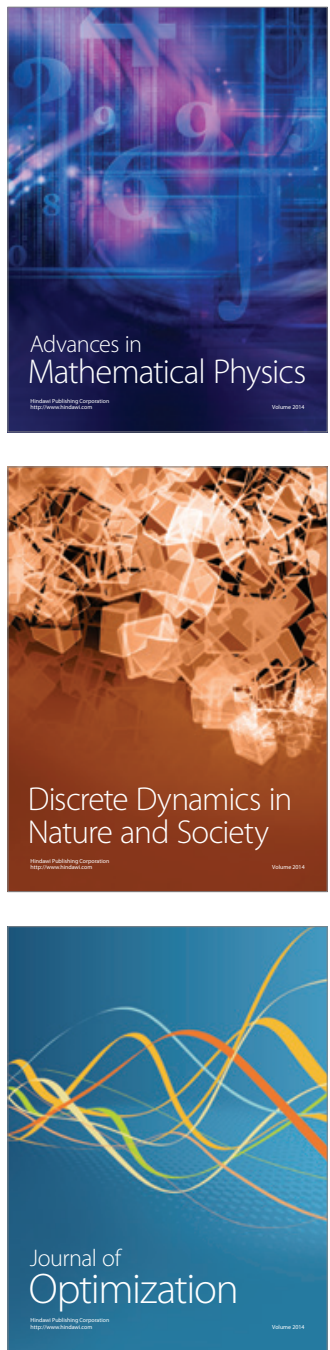